\title{
The protected areas system in Brazil as a baseline condition for wetlands management and fish conservancy: the example of the Pantanal National Park
}

\author{
Carla N. M. Polaz ${ }^{1,2}$, Fabio C. Ferreira ${ }^{3}$ and Miguel Petrere Júnior ${ }^{4}$
}

Considering the need for the Brazilian government to develop tools for environmental monitoring for biodiversity conservancy purposes in the national protected areas system, this paper focuses on determining reference site metrics (or baselines) for adapting the Index of Biotic Integrity (IBI) based on the fish assemblages in the Pantanal National Park (PNP). The habitats in the PNP were grouped into four categories: main rivers, corixos (channels connecting the floodplain), permanent bays, and temporary bays. Fish samplings were performed at 12 points during the dry season (Oct-Nov 2010 and 2011). 146 fish species were identified from the total 18,954 individuals collected with standardized fishing gear. There was no association between the structure of the fish assemblage and categories, suggesting a theory on homogeneity of habitats. The final $\mathrm{IBI}_{\mathrm{PNP}}$ consists of nine metrics, most of them were framed in excellent class, some in fair, and none in poor. There was no significant difference in $\mathrm{IBI}_{\mathrm{PNP}}$ scores between the two sampled years. This approach provides a direct application for wetland management purposes.

Keywords: Conservation unit, Fish assemblages, Floodplain, Index of biotic integrity, Multimetric indices.

É iminente que o Brasil desenvolva ferramentas para monitorar o sistema de áreas protegidas do país, formado especialmente pelas Unidades de Conservação e tendo como foco a proteção efetiva da biodiversidade. Nesse contexto, esse trabalho discute métricas de referência (ou linhas de base) para compor um Índice de Integridade Biótica (IIB) baseado nas comunidades de peixes, tendo como área de estudo o Parque Nacional do Pantanal Matogrossense. Os hábitats encontrados no Parque foram agrupados em quatro estratos ambientais: rios, corixos (cursos d'água que se conectam à planície de inundação) e baías permanentes e temporárias. As coletas de peixes foram realizadas em 12 pontos amostrais durante o período seco (out/nov de 2010 e 2011). Foram identificadas 146 espécies de peixes, totalizando 18.954 exemplares coletados em amostragens padronizadas. Não houve associação entre a estrutura da comunidade e os estratos ambientais, sugerindo homogeneidade de hábitats. O IIB final foi composto por nove métricas, a maior parte alocada na classe excelente de integridade biótica, poucas na classe regular e nenhuma na classe pobre. Não houve diferença significativa no IIB entre os dois anos consecutivos. Esses resultados oferecem subsídios técnicos para a tomada de decisão sobre a gestão das áreas alagáveis no Pantanal brasileiro.

Palavras-chave: Assembleia de peixes, Índice de integridade biótica, Índices multimétricos, Planície de inundação, Unidade de conservação.

\section{Introduction}

Human disturbances interact in a complex way with aquatic ecosystems, and their effects can rarely be evaluated using only physical or chemical variables as indirect measures of environmental integrity. Assessing the response of the aquatic biota (e.g., freshwater fish) often provides a more integrated view of environmental impacts (Fausch et al., 1990). These are the principles of the original idea of the Index of Biotic Integrity (IBI) (Karr, 1981; Karr et al., 1986) that combined 12 metrics (or attributes) of fish assemblages to measure and predict the outcomes of anthropogenic environmental disturbance through a given environmental gradient.

\footnotetext{
'Programa de Pós-Graduação em Ciências da Engenharia Ambiental, Escola de Engenharia de São Carlos, Centro de Recursos Hídricos e Ecologia Aplicada, Universidade de São Paulo, Rod. Domingos Innocentini, km 13, 292, São Carlos, São Paulo, Brasil. ${ }^{2}$ Centro Nacional de Pesquisa e Conservação da Biodiversidade Aquática Continental, Instituto Chico Mendes de Conservação da Biodiversidade, Rod. Pref. E. Nemésio Pereira de Godoy (SP 201) km 6.5, 64, Pirassununga, São Paulo, Brazil.carlapolaz@gmail.com (corresponding author)

${ }^{3}$ Departamento de Ciências do Mar, Campus Baixada Santista, Universidade Federal de São Paulo, Avenida Almirante Saldanha da Gama, 89, Ponta da Praia, 11030-400, Santos, São Paulo, Brazil. fabiocferreira@gmail.com

${ }^{4}$ Programa de Pós-Graduação em Sustentabilidade de Ecossistemas Costeiros e Marinhos, UNISANTA, Rua Oswaldo Cruz, 277 (Boqueirão), 11045-907, Santos, São Paulo, Brazil. mpetrerejr@gmail.com
} 
The applications of the IBI in the U.S. and Europe demonstrated the effectiveness of the method and highlighted the need to modify or adapt the index to particularities of regions and systems under study (Hughes, Oberdorff, 1999; Simon, Sanders, 1999; Roset et al., 2007).

In Brazil, the first adaptation of the IBI based on fish assemblages was carried out in 1998 (Araújo et al., 2003) in one of the most critical segments of the highly polluted Paraíba do Sul River, which runs through the main industrial region of the country. In the Upper Paraná River, small streams (Ferreira, Casatti, 2006; Casatti et al., 2009) and reservoirs (Petesse et al., 2007; Terra et al., 2010) were also investigated by adapting the IBI to these systems.

According to the original proposition (Karr, 1981; Karr et al., 1986), the intensity of environmental degradation should be evaluated by comparing the modified habitats with pristine environments in the same or a nearby region (such as a protected area) taken as reference conditions or baselines. However, pristine sites are frequently not found, explained by an environmental and cultural "illness" known as SBS - Shifting Baseline Syndrome (Pauly, 1995). In those cases, some authors have considered the site with the lowest degree of modification to represent reference conditions (Hughes et al., 1986; McDonough, Hickman, 1999; Pont et al., 2006). From this moment on, the baseline becomes shifted in a contagious and probably irreversible process. One generation later (25-30 yrs.), there will last no environmental collective memory of the prior baseline.

In the face of rapid degradation of aquatic environments around the world, it is argued that adaptations of IBI should also be undertaken for the few environments where human modifications are still not apparent. For these environments, reference conditions can and should be established before human modification becomes evident and irreversible. This idea is, again, in line with the SBS concept: after an anthropogenic impacts are disseminated, there are no reference points that could be used as a base for future evaluation of ongoing stresses on ecosystem functionality (Pauly, 1995). The problem arises because there are few situations in which a monitoring tool is implemented before the impact is present. Nonetheless, efforts should be made to change this scenario, at least for the few ecosystems that still have pristine conditions, so that the reference point could drive policy makers in proposing environmental and conservation programs.

Thus, the present study aims to adapt the IBI for the fish community of the Pantanal National Park, a large wetland area in Brazil. The most remarkable characteristic of wetlands of the Pantanal is seasonal variation in water levels, which controls ecosystem dynamics. The Pantanal is a Biosphere Reserve by UNESCO and can be considered a biodiversity refuge among Brazilian wetlands.

\section{Material and Methods}

Study area. The Pantanal National Park (PNP) is located in the extreme southwest of the Mato Grosso State in the municipality of Poconé at the junction of the Paraguay and Cuiabá rivers (Fig. 1). This unique Park retains a considerable sample of the original lowland with a total area of 1,350 $\mathrm{km}^{2}$, equivalent to $1 \%$ of the Brazilian Pantanal biome (Instituto Brasileiro do Meio Ambiente e dos Recursos Naturais Renováveis, 2003). Founded in September 1981, this national protected area (Ministério do Meio Ambiente, 2000) has been managed since 2007 by the Instituto Chico Mendes de Conservação da Biodiversidade, an agency of the Brazilian government.

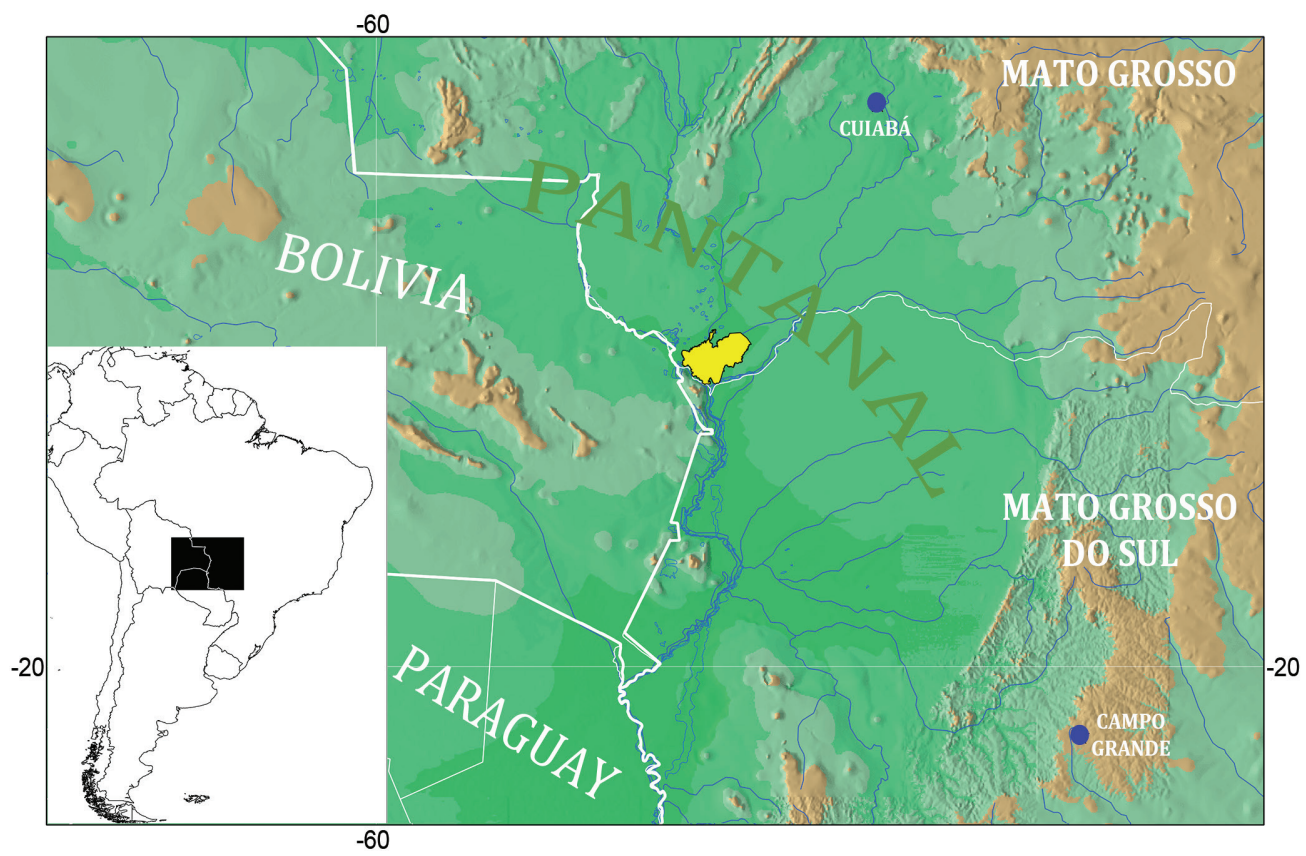

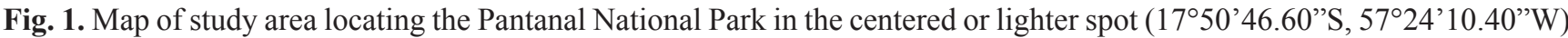
of the Upper Paraguay River basin, Brazil. 
Sampling design and fish collection. Based on cartographic charts of habitat distribution and photographic records, four regions, totaling 12 sampling sites (named P1 through P12), were pre-established and visited in the field for confirmation. Strata were defined based on water flow (lotic or lentic) and connectivity with main river channel (permanent or temporary connection). Category A (lotic environments represented by $\mathrm{P} 4$ and P6) was characterized by two major rivers, Cuiabá and Paraguay, in the Pantanal floodplain. These rivers are permanent water bodies with well-defined channels, and, henceforth, this category is designated as main channel sites. Category B (lentic environments; P3, P7, and P8) is composed of floodplain lakes with permanent connection to the main river channel throughout the hydrological cycle (Burro, Três Bocas, and Morro bays). This category is identified as permanent bays. With the exception of Três Bocas Bay, which is deeper, the other two are shallower and are about $50 \mathrm{~cm}$ deep during the dry season. Category $\mathrm{C}$ (lentic environments; $\mathrm{P} 5, \mathrm{P} 10$, and $\mathrm{P} 11$ ) is represented by smaller bays (Nove, Morro, and Biguerinho river bays), called baiotas, with intermittent connection with the main river and average depths ranging from 1 to 2 meters during the high-water season. These river bays can dry out completely in the low water season (except in years with higher flows) and, hereinafter, are referred to as temporary bays. Lastly, category D (lotic environments; P1, P2, P9, and P12) is represented by connected channels linking the main rivers and other elements of the floodplain, such as bays, and are referred locally as corixos (Boca do Ricardo, Morro do Caracara, Caracarazinho do Morro river, and Biguerinho river). As they link the main rivers to the floodplain, the current is weaker than in the main channel, and they have no delimited headwater and mouth. However, they may exhibit very well defined channels. When the channels are not well defined, they are called vazante (ebb), where the water simply overflows. This type of body is identified as corixos.

In a preliminary recognition campaign in the PNP area (Sep-2009), these 12 sampling points were selected out of 40 other potential locations based on: a) typology of habitat structure, b) cartographic analysis, and c) access by boat, considering that the core area of the PNP is unreachable. The samples were taken during the dry season of 2010 and 2011 because, during wet season, the four categories are indistinguishable and fish capture is less efficient due to high water level.

The sampling effort was characterized by 30 sequential hauls, separated by a minimum distance of $3 \mathrm{~m}$ along the marginal vegetation. Medium to large fish were collected with two cast nets (with 2 and $8 \mathrm{~cm}$ between opposite knots and with 15 hauls of each cast net in each sampling point) and gillnets (set of 7 nets with mesh ranging from 4 to 16 $\mathrm{cm}$ between opposite knots that were set for 2 hours in the water during sunlight). The sequence of nets was set at random. The small-sized fish species, usually associated with macrophytes, were collected with sieves built from net shading (nylon $1.5 \mathrm{~mm}$ opposite knots mesh size) that had dimensions of $1.5 \mathrm{~m} \mathrm{x} 1.5 \mathrm{~m}$ and $0.7 \mathrm{~m}$ depth.

Metrics selection and description. The $\mathrm{IBI}_{\mathrm{PNP}}$ metrics were adapted from Karr et al. (1986), Ganasan, Hughes (1998), Araújo et al. (2003), Bozzetti, Schulz (2004), Petesse et al. (2007), and Ferreira (2011). 34 candidate metrics were selected and grouped into four categories (Tab. 1) considering the following traits of the fish community structure: (i) richness and species assemblages (8 metrics); (ii) fish abundance ( 8 metrics); (iii) composition of feeding groups (10 metrics) and; (iv) indicators of environmental health at community level (8 metrics). All metrics were calculated in numbers of fish, as the measurement of biomass is difficult to obtain in the field due to the amount of fish collected. With exception of metrics 32 (number of introduced species) and 33 (number of hybrid species) only native species of the Pantanal ecosystem were considered.

In order to select appropriate metrics to compose the $\mathrm{IBI}_{\mathrm{PNP}}, 3$ tests were conducted (i) a range test to assess the magnitude and variability of the metrics in the sampling sites, (ii) a responsiveness test to evaluate the response of each metric to habitat modification, and (iii) a redundancy test to exclude highly correlated metrics of modification (Stoddard et al., 2008; Petesse et al., 2014). Metrics with ranges equal or smaller than 4 or with $75 \%$ of identical values (range test) were excluded, since they would be unable to detect differences among the sampling locations (Whittier et al., 2007) and therefore would have low discriminatory power. The metric responses to environmental modification (responsiveness test) were verified by the Spearman correlation coefficient $\left(\mathrm{r}_{\mathrm{S}}\right)$ between each remaining metric and the following abiotic characteristics: macrophyte cover-MC, macrophyte integrity-MI, presence of communal bird nests-PN, air temperature-AT, water temperature-WT, water transparency-WTp, depth-D, electrical conductivity-EC, dissolved oxygen-DO, and oxidation/reduction potential-ORP (Polaz, 2013). Metrics that showed significant correlation $(\mathrm{p}<0.05)$ passed the test. The Spearman correlation was also calculated to identify pairs of redundant metrics (redundancy test). For pairs of metrics that had a correlation coefficient above 0.7 (Whittier et al., 2007), the ones that exhibited the lowest number of significant correlations in the responsiveness test were excluded (McCormick et al., 2001; Whittier et al., 2007).

Criteria for scoring and classes of integrity. As in most applications of IBI, ordinal scores were used to standardize metrics in different scales considering the categories: impacted biota (1), fair or intermediate (3), and not impaired (5).

The choice of a scale with discrete values $(1,3$, and 5$)$ is mainly justified by facilitating the communication of results to non-specialists that is a desirable feature for the management of protected areas open to the public. 
Tab. 1. Candidate metrics, expected responses to disturbance and results of criteria tests (1,2 and 3$)$ to compose the IBI $I_{\mathrm{PNP}^{*}}$ The sign (-) indicates that the measure did not pass the test.

\begin{tabular}{|c|c|c|c|c|}
\hline Metrics & $\begin{array}{l}\text { Response to } \\
\text { disturbance }\end{array}$ & $\begin{array}{c}\text { Test } 1 \text { - } \\
\text { Range test }\end{array}$ & $\begin{array}{c}\text { Test } 2 \text { - } \\
\text { Responsiveness }\end{array}$ & $\begin{array}{c}\text { Test } 3 \text { - } \\
\text { Redundancy }\end{array}$ \\
\hline \multicolumn{5}{|c|}{ Richness and species composition (8 metrics) } \\
\hline 2. Total number of native fish orders (Ord) & Decrease & - & - & - \\
\hline 3. Total number of native fish family (Fam) & Decrease & Passed the test & Passed the test & Passed the test \\
\hline 4. Total number of rare fish species (Rar) & Decrease & Passed the test & - & - \\
\hline 6. Total number of Siluriformes species (SSi) & Decrease & Passed the test & Passed the test & Passed the test \\
\hline 7. Total number of Gymnotiformes species (SGy) & Decrease & Passed the test & - & - \\
\hline 8. Total number of Cichlidae species ( $\mathrm{SCi}$ ) & Decrease & Passed the test & Passed the test & Passed the test \\
\hline \multicolumn{5}{|c|}{ Abundance ( 8 metrics) } \\
\hline 12. Percent abundance of Siluriformes (NSi) & Decrease & Passed the test & Passed the test & - \\
\hline 13. Percent abundance of Gymnotiformes (NGy) & Decrease & Passed the test & Passed the test & Passed the test \\
\hline 14. Percent abundance of Cichlidae (NCi) & Decrease & Passed the test & - & - \\
\hline 15. Percent abundance of individuals larger than $30 \mathrm{~cm}$ (L30) & Decrease & Passed the test & - & - \\
\hline 16. Percent abundance of large migratory fish species (Mig) & Decrease & Passed the test & - & - \\
\hline \multicolumn{5}{|c|}{ Feeding groups (10 metrics) } \\
\hline 17. Number of carnivorous species (SCar) & Decrease & Passed the test & - & - \\
\hline 18. Percent abundance of carnivores (NCar) & Decrease & Passed the test & - & - \\
\hline 19. Number of omnivorous species (SOni) & Increase & Passed the test & - & - \\
\hline 26. Percent abundance of herbivores (NHer) & Decrease & Passed the test & - & - \\
\hline \multicolumn{5}{|c|}{ Assemblage indicators and environmental health ( 8 metrics) } \\
\hline 27. Shannon diversity índex $\left(\mathrm{H}^{\prime}\right)$ & Decrease & Passed the test & - & - \\
\hline 28. Pielou's evenness index $(\mathrm{J})$ & Decrease & Passed the test & Passed the test & Passed the test \\
\hline 29. Percent abundance of Abramites hypselonotus (A.hyp) & Decrease & Passed the test & - & - \\
\hline 30. Percent abundance of the genus Eigenmannia (Eig) & Decrease & Passed the test & - & - \\
\hline 31. Percent abundance of the genera Pygocentrus and Serrasalmus & Increase & Passed the test & - & - \\
\hline 32. Total number of introduced species (aliens/exotic/aloctonous) (SInt) & Increase & - & - & - \\
\hline 33. Total number of hybrid species (NHib) & Increase & - & - & - \\
\hline 34. Percent abundance of fish with anomalies (damage/deformation) (NAno) & Increase & - & - & - \\
\hline
\end{tabular}

In order to exclude the effect of outliers, only the interval between the $5^{\text {th }}$ and $95^{\text {th }}$ percentiles were considered. For the remaining metrics, the $25^{\text {th }}$ percentile of the distribution as the inferior limit was assumed. Values above the $25^{\text {th }}$ percentile were considered unimpaired (score 5), and values below the $25^{\text {th }}$ percentile were bisected. Those in the top half received a score of 3 , and those in the bottom half received a score of 1 . Gerritsen et al. (2003) recommended this procedure for scoring when most sites are undisturbed. This score attribution was inverted for metrics that increase with disturbance intensity such as the number of introduced species. Finally, the scores were summed to obtain the final IBI, which was classified as "poor", "fair", or "excellent" classes of biotic integrity. A two-way Analysis of Variance was used to test the effects of water body categorization and year of sampling on the final $\mathrm{IBI}_{\mathrm{PNP}}$ (Zar, 2010). 


\section{Results}

Fish assemblage composition. 18,954 individuals were collected and distributed in nine orders for 31 families and 146 species of freshwater fish (Britski et al., 2007; Oliveira et al., 2011; Eschmeyer et al., 2017) (Tab. 2). Some of these species are listed in Polaz et al. (2014). The phylogenetic orders comprising the highest percentage of the richness were Characiformes (69 species; 47.3\%) and Siluriformes (50 species; $34.2 \%$ ), followed by Perciformes (11 species; $7.5 \%$ ), and Gymnotiformes (11 species; 7.5\%). These four orders represented $96.5 \%$ of the total fish assemblages. The highest number of species was within the Characidae family $(25.3 \%)$, followed by Loricariidae (10.3\%) and Cichlidae (6.8\%).

Tab. 2. Taxonomic list of sampled species according to Eschmeyer, Fricke (2017) and species abundance, feeding and migratory guilds in the region sampled in the Pantanal National Park, during the dry season (Oct-Nov, 2010 and 2011). PB permanent bays, TB - temporary bays, CR - "corixos", MR - main rivers. TG - trophic guilds; C - carnivores; I - insectivores/ invertivores; O - omnivores; D - detritivores; H - herbivores; ND - not determined trophic guild; LdM - long-distance migratory species.

\begin{tabular}{|c|c|c|c|c|c|c|c|c|}
\hline ID & SPECIES & PB & TB & $\mathrm{CR}$ & MR & Total & TG & LdM \\
\hline & ACTINOPTERYGII & & & & & & & \\
\hline & BELONIFORMES & & & & & & & \\
\hline & Belonidae & & & & & & & \\
\hline \multirow[t]{3}{*}{1} & Potamorhaphis eigenmanni Miranda Ribeiro, 1915 & 0 & 1 & 1 & 0 & 2 & $\mathrm{C}$ & \\
\hline & CHARACIFORMES & & & & & & & \\
\hline & Acestrorhynchidae & & & & & & & \\
\hline \multirow[t]{2}{*}{2} & Acestrorhynchus pantaneiro Menezes, 1992 & 0 & 1 & 4 & 0 & 5 & $\mathrm{C}$ & \\
\hline & Anostomidae & & & & & & & \\
\hline 3 & Abramites hypselonotus (Günther, 1868) & 44 & 9 & 24 & 13 & 90 & $\mathrm{H}$ & \\
\hline 4 & Leporinus friderici Bloch, 1794 & 1 & 0 & 6 & 0 & 7 & $\mathrm{O}$ & \\
\hline 5 & Leporinus lacustris Amaral Campos, 1945 & 9 & 22 & 16 & 6 & 53 & $\mathrm{O}$ & \\
\hline 6 & Leporinus striatus Kner, 1858 & 13 & 1 & 8 & 11 & 33 & $\mathrm{O}$ & \\
\hline 7 & Megaleporinus macrocephalus (Garavello \& Britski, 1988) & 1 & 0 & 7 & 1 & 9 & $\mathrm{O}$ & Yes \\
\hline 8 & Megaleporinus obtusidens (Valenciennes, 1837) & 0 & 1 & 2 & 0 & 3 & $\mathrm{O}$ & \\
\hline 9 & Schizodon borellii (Boulenger, 1900) & 8 & 12 & 42 & 7 & 69 & $\mathrm{H}$ & \\
\hline \multirow[t]{2}{*}{10} & Schizodon isognathus Kner, 1858 & 2 & 0 & 1 & 0 & 3 & $\mathrm{H}$ & \\
\hline & Bryconidae & & & & & & & \\
\hline \multirow[t]{2}{*}{11} & Brycon hilarii (Valenciennes, 1850) & 0 & 1 & 1 & 0 & 2 & $\mathrm{O}$ & Yes \\
\hline & Characidae & & & & & & & \\
\hline 12 & Aphyocharax anisitsi Eigenmann \& Kennedy, 1903 & 0 & 12 & 0 & 0 & 12 & I & \\
\hline 13 & Aphyocharax dentatus Eigenmann \& Kennedy, 1903 & 4 & 27 & 16 & 2 & 49 & $\mathrm{O}$ & \\
\hline 14 & Aphyocharax nattereri (Steindachner, 1882) & 18 & 108 & 45 & 2 & 173 & I & \\
\hline 15 & Aphyocharax rathbuni Eigenmann, 1907 & 0 & 34 & 3 & 0 & 37 & I & \\
\hline 16 & Astyanax abramis (Jenyns, 1842) & 0 & 68 & 0 & 1 & 69 & I & \\
\hline 17 & Astyanax asuncionensis Géry, 1972 & 1 & 3 & 2 & 0 & 6 & $\mathrm{O}$ & \\
\hline 18 & Bryconamericus exodon Eigenmann, 1907 & 15 & 0 & 21 & 63 & 99 & I & \\
\hline 19 & Bryconamericus stramineus Eigenmann, 1908 & 25 & 16 & 47 & 4 & 92 & I & \\
\hline 20 & Charax leticiae Lucena, 1987 & 1 & 20 & 1 & 1 & 23 & $\mathrm{C}$ & \\
\hline 21 & Ctenobrycon alleni (Eigenmann \& Kennedy, 1907) & 18 & 55 & 45 & 1 & 119 & $\mathrm{O}$ & \\
\hline 22 & Cynopotamus kincaidi (Schultz, 1950) & 1 & 0 & 1 & 0 & 2 & $\mathrm{C}$ & \\
\hline 23 & Galeocharax humeralis (Valenciennes, 1834) & 6 & 8 & 2 & 0 & 16 & $\mathrm{C}$ & \\
\hline 24 & Gymnocorymbus ternetzi (Boulenger, 1895) & 1 & 1 & 0 & 0 & 2 & $\mathrm{O}$ & \\
\hline 25 & Hemigrammus lunatus Durbin, 1918 & 331 & 284 & 114 & 19 & 748 & $\mathrm{O}$ & \\
\hline 26 & Hemigrammus marginatus Ellis, 1911 & 0 & 34 & 5 & 0 & 39 & I & \\
\hline 27 & Hyphessobrycon eques (Steindachner, 1882) & 50 & 294 & 116 & 23 & 483 & I & \\
\hline 28 & Hyphessobrycon megalopterus (Eigenmann, 1915) & 4 & 74 & 50 & 0 & 128 & $\mathrm{O}$ & \\
\hline 29 & Jupiaba acanthogaster (Eigenmann, 1911) & 218 & 205 & 80 & 30 & 533 & $\mathrm{O}$ & \\
\hline 30 & Moenkhausia bonita Benine, Castro \& Sabino, 2004 & 1 & 14 & 5 & 0 & 20 & $\mathrm{O}$ & \\
\hline 31 & Moenkhausia dichroura $($ Kner, 1858) & 38 & 17 & 37 & 20 & 112 & $\mathrm{O}$ & \\
\hline
\end{tabular}


Tab. 2. (continued)

\begin{tabular}{|c|c|c|c|c|c|c|c|c|}
\hline ID & SPECIES & $\mathrm{PB}$ & TB & $\mathrm{CR}$ & MR & Total & TG & LdM \\
\hline 32 & Moenkhausia forestii Benine, Mariguela \& Oliveira, 2009 & 46 & 190 & 45 & 10 & 291 & $\mathrm{O}$ & \\
\hline 33 & Moenkhausia oligolepis (Günther, 1864) & 1 & 0 & 0 & 0 & 1 & $\mathrm{O}$ & \\
\hline 34 & Odontostilbe paraguayensis Eigenmann \& Kennedy, 1903 & 102 & 0 & 30 & 0 & 132 & $\mathrm{O}$ & \\
\hline 35 & Odontostilbe pequira (Steindachner, 1882) & 224 & 71 & 83 & 46 & 424 & $\mathrm{O}$ & \\
\hline 36 & Poptella paraguayensis (Eigenmann, 1907) & 5 & 3 & 5 & 0 & 13 & I & \\
\hline 37 & Prionobrama paraguayensis (Eigenmann, 1914) & 298 & 854 & 336 & 22 & 1510 & $\mathrm{O}$ & \\
\hline 38 & Psellogrammus kennedyi (Eigenmann, 1903) & 7 & 16 & 105 & 10 & 138 & $\mathrm{O}$ & \\
\hline 39 & Roeboides descalvadensis Fowler, 1932 & 0 & 14 & 5 & 3 & 22 & $\mathrm{C}$ & \\
\hline 40 & Roeboides microlepis (Reinhardt, 1851) & 1 & 1 & 5 & 7 & 14 & $\mathrm{C}$ & \\
\hline 41 & Serrapinnus calliurus (Boulenger, 1900) & 403 & 323 & 108 & 55 & 889 & $\mathrm{O}$ & \\
\hline 42 & Serrapinnus kriegi (Schindler, 1937) & 0 & 0 & 24 & 0 & 24 & $\mathrm{O}$ & \\
\hline 43 & Serrapinnus microdon (Eigenmann, 1915) & 53 & 1005 & 395 & 0 & 1450 & $\mathrm{O}$ & \\
\hline \multirow[t]{2}{*}{44} & Tetragonopterus argenteus Cuvier, 1816 & 3 & 3 & 10 & 0 & 16 & $\mathrm{O}$ & \\
\hline & Crenuchidae & & & & & & & \\
\hline 45 & Characidium laterale (Boulenger, 1895) & 150 & 487 & 306 & 72 & 1015 & I & \\
\hline \multirow[t]{2}{*}{46} & Characidium aff. zebra Eigenmann, 1909 & 8 & 0 & 2 & 27 & 37 & I & \\
\hline & Curimatidae & & & & & & & \\
\hline 47 & Curimatella dorsalis (Eigenmann \& Eigenmann, 1889) & 0 & 5 & 0 & 0 & 5 & $\mathrm{D}$ & \\
\hline 48 & Curimatopsis myersi Vari, 1982 & 0 & 96 & 2 & 0 & 98 & $\mathrm{D}$ & \\
\hline 49 & Cyphocharax gillii (Eigenmann \& Kennedy, 1903) & 0 & 6 & 2 & 4 & 12 & $\mathrm{D}$ & \\
\hline 50 & Potamorhina squamoralevis (Braga \& Azpelicueta, 1983) & 27 & 37 & 57 & 20 & 141 & $\mathrm{D}$ & \\
\hline \multirow[t]{2}{*}{51} & Psectrogaster curviventris Eigenmann \& Kennedy, 1903 & 3 & 20 & 63 & 1 & 87 & $\mathrm{D}$ & \\
\hline & Cynodontidae & & & & & & & \\
\hline \multirow[t]{2}{*}{52} & Rhaphiodon vulpinus Spix \& Agassiz, 1829 & 6 & 0 & 1 & 2 & 9 & $\mathrm{C}$ & \\
\hline & Erythrinidae & & & & & & & \\
\hline 53 & Hoplerythrinus unitaeniatus (Spix \& Agassiz, 1829) & 2 & 0 & 1 & 0 & 3 & $\mathrm{O}$ & \\
\hline \multirow[t]{2}{*}{54} & Hoplias aff. malabaricus (Bloch, 1794) & 12 & 5 & 16 & 9 & 42 & $\mathrm{C}$ & \\
\hline & Gasteropelecidae & & & & & & & \\
\hline 55 & Gasteropelecus sternicla (Linnaeus, 1758) & 7 & 7 & 50 & 1 & 65 & I & \\
\hline \multirow[t]{2}{*}{56} & Thoracocharax stellatus (Kner, 1858) & 0 & 0 & 0 & 5 & 5 & I & \\
\hline & Hemiodontidae & & & & & & & \\
\hline \multirow[t]{2}{*}{57} & Hemiodus orthonops Eigenmann \& Kennedy, 1903 & 1 & 0 & 0 & 1 & 2 & $\mathrm{O}$ & \\
\hline & Iguanodectidae & & & & & & & \\
\hline \multirow[t]{2}{*}{58} & Piabucus melanostoma Holmberg, 1891 & 10 & 0 & 5 & 0 & 15 & $\mathrm{H}$ & \\
\hline & Lebiasinidae & & & & & & & \\
\hline \multirow[t]{2}{*}{59} & Pyrrhulina australis Eigenmann \& Kennedy, 1903 & 45 & 187 & 86 & 17 & 335 & I & \\
\hline & Prochilodontidae & & & & & & & \\
\hline \multirow[t]{2}{*}{60} & Prochilodus lineatus (Valenciennes, 1837) & 26 & 8 & 1 & 2 & 37 & $\mathrm{D}$ & Yes \\
\hline & Serrassalmidae & & & & & & & \\
\hline 61 & Metynnis maculatus (Kner, 1858) & 0 & 2 & 0 & 0 & 2 & $\mathrm{H}$ & \\
\hline 62 & Myleus levis Eigenmann \& McAtee, 1907 & 0 & 4 & 11 & 1 & 16 & $\mathrm{H}$ & \\
\hline 63 & Mylossoma duriventre (Cuvier, 1818) & 6 & 7 & 25 & 11 & 49 & $\mathrm{H}$ & \\
\hline 64 & Piaractus mesopotamicus (Holmberg, 1887) & 4 & 1 & 9 & 2 & 16 & $\mathrm{H}$ & Yes \\
\hline 65 & Pygocentrus nattereri Kner, 1858 & 62 & 36 & 65 & 17 & 180 & $\mathrm{C}$ & \\
\hline 66 & Serrasalmus maculatus Kner, 1858 & 9 & 56 & 25 & 4 & 94 & $\mathrm{C}$ & \\
\hline \multirow[t]{2}{*}{67} & Serrasalmus marginatus Valenciennes, 1837 & 57 & 10 & 39 & 3 & 109 & $\mathrm{C}$ & \\
\hline & Triportheidae & & & & & & & \\
\hline 68 & Engraulisoma taeniatum Castro, 1981 & 0 & 0 & 2 & 0 & 2 & I & \\
\hline 69 & Triportheus nematurus (Kner, 1858) & 9 & 7 & 23 & 6 & 45 & I & \\
\hline 70 & Triportheus pantanensis Malabarba, 2004 & 6 & 3 & 27 & 1 & 37 & I & \\
\hline
\end{tabular}


Tab. 2. (continued)

\begin{tabular}{|c|c|c|c|c|c|c|c|c|}
\hline ID & SPECIES & $\mathrm{PB}$ & TB & $\mathrm{CR}$ & MR & Total & TG & LdM \\
\hline & CLUPEIFORMES & & & & & & & \\
\hline & Engraulidae & & & & & & & \\
\hline \multirow[t]{3}{*}{71} & Anchoviella sp. & 0 & 20 & 4 & 0 & 24 & ND & \\
\hline & ORDER CYPRINODONTIFORMES & & & & & & & \\
\hline & Poeciliidae & & & & & & & \\
\hline \multirow[t]{2}{*}{72} & Pamphorichthys hasemani (Henn, 1916) & 0 & 0 & 1 & 0 & 1 & I & \\
\hline & Cynolebiidae & & & & & & & \\
\hline \multirow[t]{3}{*}{73} & Melanorivulus punctatus (Boulenger, 1895) & 0 & 0 & 4 & 0 & 4 & I & \\
\hline & ORDER GYMNOTIFORMES & & & & & & & \\
\hline & Apteronotidae & & & & & & & \\
\hline 74 & Apteronotus albifrons (Linnaeus, 1766) & 7 & 3 & 4 & 0 & 14 & I & \\
\hline 75 & Apteronotus caudimaculosus de Santana, 2003 & 8 & 15 & 18 & 8 & 49 & $\mathrm{O}$ & \\
\hline \multirow[t]{2}{*}{76} & Sternarchorhynchus curvirostris (Boulenger, 1887) & 0 & 0 & 2 & 0 & 2 & I & \\
\hline & Gymnotidae & & & & & & & \\
\hline 77 & Gymnotus inaequilabiatus (Valenciennes, 1839) & 7 & 7 & 5 & 0 & 19 & I & \\
\hline \multirow[t]{2}{*}{78} & Gymnotus paraguensis Albert \& Crampton, 2003 & 0 & 1 & 6 & 1 & 8 & I & \\
\hline & Hypopomidae & & & & & & & \\
\hline \multirow[t]{2}{*}{79} & Brachyhypopomus spp. & 60 & 96 & 53 & 25 & 234 & ND & \\
\hline & Ramphichthyidae & & & & & & & \\
\hline 80 & Gymnorhamphichthys britskii Carvalho, Ramos \& Albert, 2011 & 2 & 5 & 1 & 2 & 10 & I & \\
\hline \multirow[t]{2}{*}{81} & Rhamphichthys hahni (Meiken, 1937) & 0 & 1 & 0 & 1 & 2 & I & \\
\hline & Sternopygidae & & & & & & & \\
\hline 82 & Eigenmannia trilineata López \& Castello, 1966 & 960 & 638 & 180 & 323 & 2101 & I & \\
\hline 83 & Eigenmannia virescens (Valenciennes, 1836) & 22 & 29 & 126 & 9 & 186 & I & \\
\hline \multirow[t]{3}{*}{84} & Sternopygus macrurus (Bloch \& Schneider, 1801) & 83 & 68 & 61 & 36 & 248 & I & \\
\hline & ORDER PERCIFORMES & & & & & & & \\
\hline & Cichlidae & & & & & & & \\
\hline 85 & Aequidens plagiozonatus Kullander, 1984 & 5 & 0 & 10 & 0 & 15 & $\mathrm{O}$ & \\
\hline 86 & Apistogramma borellii (Regan, 1906) & 20 & 9 & 45 & 0 & 74 & I & \\
\hline 87 & Apistogramma commbrae (Regan, 1906) & 3 & 4 & 3 & 2 & 12 & I & \\
\hline 88 & Apistogramma trifasciata (Eigenmann \& Kennedy, 1903) & 82 & 116 & 141 & 30 & 369 & $\mathrm{I}$ & \\
\hline 89 & Astronotus crassipinnis (Heckel, 1840) & 0 & 0 & 2 & 0 & 2 & I & \\
\hline 90 & Cichlasoma dimerus (Heckel, 1840) & 11 & 14 & 22 & 10 & 57 & $\mathrm{D}$ & \\
\hline 91 & Crenicichla lepidota Heckel, 1840 & 39 & 52 & 65 & 24 & 180 & $\mathrm{C}$ & \\
\hline 92 & Crenicichla vittata Heckel, 1840 & 1 & 0 & 0 & 0 & 1 & $\mathrm{O}$ & \\
\hline 93 & Laetacara dorsigera (Heckel, 1840) & 1 & 0 & 0 & 0 & 1 & $\mathrm{O}$ & \\
\hline \multirow[t]{2}{*}{94} & Mesonauta festivus (Heckel, 1840) & 0 & 4 & 2 & 3 & 9 & $\mathrm{H}$ & \\
\hline & Sciaenidae & & & & & & & \\
\hline \multirow[t]{3}{*}{95} & Plagioscion ternetzi Boulenger, 1895 & 6 & 0 & 1 & 2 & 9 & $\mathrm{C}$ & \\
\hline & ORDER SILURIFORMES & & & & & & & \\
\hline & Aspredinidae & & & & & & & \\
\hline 96 & Amaralia oviraptor Friel \& Carvalho, 2016 & 1 & 0 & 0 & 0 & 1 & $\mathrm{C}$ & \\
\hline \multirow[t]{2}{*}{97} & Pseudobunocephalus rugosus (Eigenmann \& Kennedy, 1903) & 3 & 1 & 0 & 0 & 4 & $\mathrm{D}$ & \\
\hline & Auchenipteridae & & & & & & & \\
\hline 98 & Auchenipterus nigripinnis (Boulenger, 1895) & 2 & 0 & 0 & 0 & 2 & $\mathrm{C}$ & \\
\hline 99 & Auchenipterus osteomystax (Miranda Ribeiro, 1918) & 3 & 0 & 1 & 0 & 4 & $\mathrm{C}$ & \\
\hline 100 & Entomocorus benjamini Eigenmann, 1917 & 3 & 2 & 0 & 0 & 5 & I & \\
\hline 101 & Tatia neivai (Ihering, 1930) & 0 & 0 & 1 & 0 & 1 & I & \\
\hline 102 & Trachelyopterus coriaceus Valenciennes, 1840 & 1 & 0 & 0 & 0 & 1 & $\mathrm{O}$ & \\
\hline 103 & Trachelyopterus galeatus (Linnaeus, 1766) & 11 & 4 & 14 & 11 & 40 & $\mathrm{O}$ & \\
\hline 104 & Trachelyopterus striatulus (Steindachner, 1877) & 0 & 0 & 3 & 3 & 6 & $\mathrm{O}$ & \\
\hline
\end{tabular}


Tab. 2. (continued)

\begin{tabular}{|c|c|c|c|c|c|c|c|c|}
\hline ID & SPECIES & PB & TB & $\mathrm{CR}$ & MR & Total & TG & LdM \\
\hline & Callichthydae & & & & & & & \\
\hline 105 & Callichthys callichthys (Linnaeus, 1758) & 7 & 0 & 3 & 0 & 10 & $\mathrm{O}$ & \\
\hline 106 & Corydoras hastatus Eigenmann \& Eigenmann, 1888 & 0 & 110 & 144 & 3 & 257 & I & \\
\hline \multirow[t]{2}{*}{107} & Hoplosternum littorale (Hancock, 1828) & 0 & 0 & 2 & 0 & 2 & I & \\
\hline & Doradidae & & & & & & & \\
\hline 108 & Anadoras weddellii (Castelnau, 1855) & 0 & 1 & 3 & 0 & 4 & $\mathrm{O}$ & \\
\hline 109 & Ossancora eigenmanni (Boulenger, 1895) & 3 & 7 & 15 & 14 & 39 & I & \\
\hline 110 & Oxydoras kneri Bleeker, 1862 & 13 & 0 & 12 & 2 & 27 & $\mathrm{O}$ & \\
\hline 111 & Platydoras armatulus (Valenciennes, 1840) & 5 & 1 & 2 & 5 & 13 & $\mathrm{O}$ & \\
\hline \multirow[t]{2}{*}{112} & Pterodoras granulosus (Valenciennes, 1821) & 1 & 0 & 8 & 2 & 11 & $\mathrm{O}$ & \\
\hline & Heptapteridae & & & & & & & \\
\hline 113 & Imparfinis spp. & 12 & 20 & 77 & 4 & 113 & ND & \\
\hline 114 & Phenacorhamdia hoehnei (Miranda Ribeiro, 1914) & 0 & 0 & 4 & 0 & 4 & I & \\
\hline 115 & Pimelodella gracilis (Valenciennes, 1835) & 0 & 7 & 1 & 15 & 23 & $\mathrm{O}$ & \\
\hline 116 & Pimelodella mисоsa Eigenmann \& Ward, 1907 & 0 & 0 & 1 & 0 & 1 & I & \\
\hline 117 & Pimelodella notomelas Eigenmann, 1917 & 1 & 0 & 0 & 0 & 1 & I & \\
\hline \multirow[t]{2}{*}{118} & Rhamdia aff. quelen (Quoy \& Gaimard, 1824) & 3 & 1 & 1 & 2 & 7 & $\mathrm{O}$ & \\
\hline & Loricariidae & & & & & & & \\
\hline 119 & Ancistrus spp. & 3 & 0 & 0 & 0 & 3 & ND & \\
\hline 120 & Farlowella paraguayensis Retzer \& Page, 1997 & 0 & 0 & 2 & 8 & 10 & $\mathrm{D}$ & \\
\hline 121 & Hemiodontichthys acipenserinus (Kner, 1853) & 0 & 0 & 0 & 1 & 1 & I & \\
\hline 122 & Hypoptopoma inexspectatum (Holmberg, 1893) & 433 & 314 & 474 & 120 & 1341 & $\mathrm{D}$ & \\
\hline 123 & Hypostomus cochliodon Kner, 1854 & 18 & 43 & 28 & 22 & 111 & $\mathrm{D}$ & \\
\hline 124 & Hypostomus latifrons Weber, 1986 & 32 & 3 & 23 & 0 & 58 & $\mathrm{D}$ & \\
\hline 125 & Hypostomus spp. & 83 & 55 & 69 & 44 & 251 & $\mathrm{D}$ & \\
\hline 126 & Loricaria spp. & 3 & 0 & 0 & 0 & 3 & $\mathrm{D}$ & \\
\hline 127 & Loricariichthys labialis (Boulenger, 1895) & 1 & 0 & 0 & 0 & 1 & $\mathrm{D}$ & \\
\hline 128 & Loricariichthys platymetopon Isbrücker \& Nijssen, 1979 & 3 & 0 & 0 & 0 & 3 & $\mathrm{D}$ & \\
\hline 129 & Otocinclus vittatus Regan, 1904 & 488 & 462 & 347 & 116 & 1413 & $\mathrm{H}$ & \\
\hline 130 & Pterygoplichthys ambrosettii (Holmberg, 1893) & 3 & 8 & 7 & 30 & 48 & $\mathrm{D}$ & \\
\hline 131 & Rineloricaria parva (Boulenger, 1895) & 409 & 236 & 214 & 166 & 1025 & $\mathrm{D}$ & \\
\hline 132 & Spatuloricaria evansii (Boulenger, 1892) & 1 & 0 & 0 & 0 & 1 & $\mathrm{D}$ & \\
\hline \multirow[t]{2}{*}{133} & Sturisoma barbatum (Kner, 1853) & 0 & 0 & 5 & 0 & 5 & $\mathrm{D}$ & \\
\hline & Pimelodidae & & & & & & & \\
\hline 134 & Hemisorubim platyrhynchos (Valenciennes, 1840) & 0 & 0 & 1 & 1 & 2 & $\mathrm{C}$ & Yes \\
\hline 135 & Hypophthalmus edentatus Spix \& Agassiz, 1829 & 0 & 1 & 0 & 0 & 1 & $\mathrm{O}$ & \\
\hline 136 & Pimelodus argenteus Perugia, 1891 & 5 & 4 & 2 & 0 & 11 & $\mathrm{O}$ & \\
\hline 137 & Pimelodus pantaneiro Souza-Filho \& Shibatta, 2007 & 1 & 1 & 4 & 1 & 7 & $\mathrm{O}$ & \\
\hline 138 & Pseudoplatystoma corruscans (Spix \& Agassiz, 1829) & 1 & 0 & 0 & 0 & 1 & $\mathrm{C}$ & Yes \\
\hline 139 & Pseudoplatystoma reticulatum Eigenmann \& Eigenmann, 1889 & 1 & 1 & 4 & 1 & 7 & $\mathrm{C}$ & Yes \\
\hline \multirow[t]{2}{*}{140} & Sorubim lima $($ Bloch \& Schneider, 1801) & 1 & 0 & 1 & 1 & 3 & $\mathrm{C}$ & Yes \\
\hline & Scoloplacidae & & & & & & & \\
\hline 141 & Scoloplax distolothrix Schaefer, Weitzman \& Britski, 1989 & 2 & 0 & 0 & 8 & 10 & I & \\
\hline \multirow[t]{2}{*}{142} & Scoloplax empousa Schaefer, Weitzman \& Britski, 1989 & 1 & 2 & 0 & 0 & 3 & I & \\
\hline & Trichomycteridae & & & & & & & \\
\hline 143 & Ituglanis eichorniarum (Miranda Ribeiro, 1912) & 1 & 0 & 0 & 1 & 2 & I & \\
\hline 144 & Ituglanis herberti (Miranda Ribeiro, 1940) & 1 & 0 & 0 & 0 & 1 & I & \\
\hline \multirow[t]{3}{*}{145} & Trichomycterus johnsoni (Fowler, 1932) & 0 & 1 & 0 & 0 & 1 & I & \\
\hline & SYNBRANCHIFORMES & & & & & & & \\
\hline & Synbranchidae & & & & & & & \\
\hline \multirow[t]{2}{*}{146} & Synbranchus marmoratus Bloch, 1795 & 0 & 1 & 2 & 1 & 4 & I & \\
\hline & & 5280 & 7154 & 4902 & 1621 & 18954 & & \\
\hline
\end{tabular}


Five feeding groups were recognized with the most common being insectivory/invertivory (47 species; $32.2 \%$ ), followed by omnivory with 44 species $(30.1 \%)$, carnivory/piscivory (21 species, $14.4 \%$ ), detritivory (20 species, $13.7 \%$ ), and herbivory represented by 10 species $(6.9 \%)$. Four entities were considered undetermined (2.7\%): Anchoviella sp., Ancistrus spp., Brachyhypopomus spp., and Imparfinis spp. Additional information on species list and categorization into feeding and migratory guilds could be also consulted in Polaz (2013, chapter 2, p.106-8).

Metrics selection. Nine of the 34 candidate metrics passed the criteria established by the range, responsiveness, and redundancy tests (Tab. 1). The number of native orders (metric 2) ranged from 3 to 6 , and, therefore, they did not pass the range test (range $<4$ ). Introduced species or hybrids were not sampled (metrics 32 and 33, respectively), and only 4 of the 24 samples exhibited had single individual anomaly (metric 34). In all these cases, the metrics did not meet the first criteria, as more than $75 \%$ of the values were zero.

Ten of the remaining 30 metrics $(3,6,8,9,10,11,12,13$, 25 , and 28) passed the second test (responsiveness), as they were correlated with at least one environmental variable. Most metrics (3, 8, 9, 25, and 28) showed a significant correlation with only one of the abiotic factors, one metric (10) with two factors, three metrics $(6,12$, and 13) with three factors, and one metric (11) with four factors. Among the abiotic variables, the macrophyte cover (MC) and the oxidation-reduction potential (ORP) showed the highest number of metrics correlation. In particular, four metrics were correlated with $\mathrm{MC}$, while there were four others with ORP. A test was proposed for the communal bird nests (locally known as ninhais) as a metrics because, especially in the reproductive season, they can provide a relevant feeding microhabitat for the fish community. Nonetheless, this factor and water depth were not correlated with any metrics.

From the 10 metrics accepted by the second test, the pairwise correlation matrix was calculated using the Spearman correlation coefficient (see more in supplementary material), resulting in only one redundant pair (metrics 11 and 12, $\mathrm{r}_{\mathrm{s}}=-0.863$ ). It was decided to keep metric 11 as it had the greater number of significant correlations (four correlations) with the environmental variables (responsiveness test). Therefore, only metric 12 did not pass in the test of redundancy.

Metrics scoring and $\mathrm{IBI}_{\mathrm{PNP}}$ calculation. All nine selected metrics are expected to decrease their values with increasing intensities of disturbance. Therefore, lower scores indicate worse conditions, and the highest scores indicate the best conditions. As there are nine metrics, the final $\mathrm{IBI}_{\mathrm{PNP}}$ for a site could range from 9 (the worst condition in which all of the metrics are given the value 1) to 45 (the best condition in which all metrics are given the value 5) (Tab. 3).

Tab. 3. Score intervals and ranges of values of the metrics for calculating the $\mathrm{IBI}_{\mathrm{PNP}}$

\begin{tabular}{|c|c|c|c|c|c|}
\hline Metrics & 1 & 3 & 5 & Minimum & Maximum \\
\hline 3. Total number of native fish family (Fam) & $<13$ & $13-14$ & $>14$ & 11 & 23 \\
\hline 6. Total number of Siluriformes species ( $\mathrm{SSi}$ ) & $<8$ & $8-10$ & $>10$ & 6 & 18 \\
\hline 8. Total number of Cichlidae species ( $\mathrm{SCi}$ ) & $<1$ & $1-2$ & $>2$ & 0 & 6 \\
\hline 9. Total number of individuals $(\mathrm{N})$ & $<443$ & $443-769$ & $>769$ & 105 & 2519 \\
\hline 10. Number of species comprising $80 \%$ of abundance (N80) & $<7$ & $7-10$ & $>10$ & 3 & 28 \\
\hline 11. Percent abundance of Characiformes (NCh) & $<16,05$ & $16,05-27,25$ & $>27,25$ & 4,44 & 87,37 \\
\hline 13. Percent abundance of Gymnotiformes (NGy) & $<9,42$ & $9,42-15,44$ & $>15,44$ & 3,18 & 47,77 \\
\hline 25. Number of herbivorous species (SHer) & $<1$ & $1-2$ & $>2$ & 0 & 7 \\
\hline 28. Pielou's evenness index $(J)$ & $<0,56$ & $0,56-0,61$ & $>0,61$ & 0,51 & 0,86 \\
\hline
\end{tabular}

Sites with excellent biotic integrity (ranging from 35 to 45) represented the best-observed situations. The community composition includes the most intolerant forms in all ranges of age category and sex ratio. They have a diverse and balanced trophic structure, the presence of large species and migratory fish, and the expected fish diversity for the biome. Fair conditions (ranging from 22 to 34 ) are those sites in which the number of species is close the expected richness, having a relatively balanced trophic structure but with some species decreasing their relative abundance and size below the values expected for the best condition. This fish community begins to show some signs of stress and needs to be monitored. The poor sites (ranging from 9 to 21) have low diversity, simplified trophic structure, dominance of tolerant and introduced species (non-native and/or exotic ones), and may show a high percentage of individuals with anomalies and parasites.

The $\mathrm{IBI}_{\mathrm{PNP}}$ ranged from 27 to 45 . No sites were classified as poor, 19 were excellent ( $\left.\mathrm{IBI}_{\mathrm{PNP}} \geq 35\right)$, and only 5 were classified as fair ( $\mathrm{IBI}_{\mathrm{PNP}}$ between 22 and 34). In 2010, with the exception of P10 ("fair"), all sites fit in the best class of integrity ("excellent"), and in 2011, while P10 increased from "fair" to "excellent", four points (P3, P4, P5, and P9) had their scores decreased (from "excellent" to "fair") (Tab. 4).

However, there were no significant effects of water body categorization $\left(\mathrm{F}_{3,16}=1.48, \mathrm{p}=0.257\right)$, year $\left(\mathrm{F}_{2,16}=0.39, \mathrm{p}\right.$ $=0.543)$, or interaction $\left(\mathrm{F}_{3.16}=1.84, \mathrm{p}=0.180\right)$ on the mean values for the $\mathrm{IBI}_{\mathrm{PNP}}$ (Figs. 2-3). 
Tab. 4. Scores of selected metrics for $\mathrm{IBI}_{\mathrm{PNP}}$ in 24 sampling points in the Pantanal National Park during the dry period (Oct-Nov) of 2010 and 2011. (*) Metrics that had different classes of integrity in 2010 and 2011.

\begin{tabular}{|c|c|c|c|c|c|c|c|c|c|c|c|c|c|}
\hline Year & Localities & Stratum & Fam & $\mathrm{SSi}$ & $\mathrm{SCi}$ & $\mathrm{N}$ & $80 \%$ & $\mathrm{NCh}$ & NGy & SHer & $\mathrm{J}$ & Total & $\mathrm{IBI}_{\mathrm{PNP}}$ \\
\hline 2010 & $\mathrm{P} 1$ & $\mathrm{CR}$ & 5 & 3 & 5 & 5 & 3 & 5 & 1 & 5 & 3 & 35 & Excellent \\
\hline 2010 & P2 & $\mathrm{CR}$ & 5 & 5 & 5 & 3 & 1 & 5 & 3 & 5 & 5 & 37 & Excellent \\
\hline 2010 & P3 & PB & 5 & 5 & 5 & 5 & 5 & 5 & 5 & 5 & 5 & 45 & Excellent \\
\hline 2010 & P4 & MR & 5 & 5 & 5 & 3 & 5 & 5 & 5 & 5 & 5 & 43 & Excellent \\
\hline 2010 & P5 & $\mathrm{TB}$ & 5 & 5 & 5 & 1 & 5 & 5 & 5 & 5 & 1 & 37 & Excellent \\
\hline 2010 & P6 & MR & 5 & 5 & 5 & 5 & 5 & 5 & 5 & 5 & 5 & 45 & Excellent \\
\hline 2010 & P7 & PB & 5 & 5 & 5 & 3 & 5 & 5 & 3 & 5 & 5 & 41 & Excellent \\
\hline 2010 & P8 & PB & 3 & 5 & 3 & 5 & 5 & 5 & 1 & 3 & 5 & 35 & Excellent \\
\hline 2010 & P9 & $\mathrm{CR}$ & 5 & 1 & 5 & 1 & 5 & 5 & 3 & 5 & 5 & 35 & Excellent \\
\hline 2010 & P10 & $\mathrm{TB}$ & 3 & 1 & 5 & 1 & 1 & 5 & 1 & 5 & 5 & 27 & Fair \\
\hline 2010 & P11 & $\mathrm{TB}$ & 5 & 5 & 5 & 1 & 3 & 5 & 1 & 5 & 5 & 35 & Excellent \\
\hline 2010 & P12 & $\mathrm{CR}$ & 5 & 5 & 5 & 3 & 3 & 5 & 1 & 5 & 5 & 37 & Excellent \\
\hline 2011 & $\mathrm{P} 1$ & $\mathrm{CR}$ & 3 & 1 & 5 & 5 & 5 & 5 & 5 & 5 & 5 & 39 & Excellent \\
\hline 2011 & P2 & $\mathrm{CR}$ & 5 & 5 & 5 & 3 & 3 & 5 & 1 & 5 & 5 & 37 & Excellent \\
\hline 2011 & P3 & PB & 5 & 3 & 3 & 3 & 3 & 1 & 5 & 3 & 5 & 31 & Fair* \\
\hline 2011 & P4 & MR & 1 & 1 & 1 & 1 & 3 & 5 & 5 & 5 & 5 & 27 & Fair* \\
\hline 2011 & P5 & $\mathrm{TB}$ & 3 & 3 & 5 & 5 & 5 & 1 & 5 & 1 & 5 & 33 & Fair* \\
\hline 2011 & P6 & MR & 5 & 3 & 5 & 3 & 5 & 5 & 5 & 5 & 5 & 41 & Excellent \\
\hline 2011 & P7 & PB & 5 & 5 & 5 & 3 & 5 & 5 & 5 & 3 & 5 & 41 & Excellent \\
\hline 2011 & P8 & PB & 5 & 5 & 5 & 3 & 3 & 5 & 5 & 5 & 5 & 41 & Excellent \\
\hline 2011 & P9 & $\mathrm{CR}$ & 5 & 1 & 5 & 1 & 3 & 5 & 3 & 5 & 5 & 33 & Fair* \\
\hline 2011 & P10 & $\mathrm{TB}$ & 5 & 3 & 5 & 5 & 3 & 5 & 1 & 5 & 5 & 37 & Excellent* \\
\hline 2011 & P11 & $\mathrm{TB}$ & 3 & 1 & 5 & 3 & 5 & 5 & 3 & 5 & 5 & 35 & Excellent \\
\hline 2011 & P12 & $\mathrm{CR}$ & 5 & 5 & 5 & 5 & 5 & 5 & 3 & 5 & 5 & 43 & Excellent \\
\hline
\end{tabular}

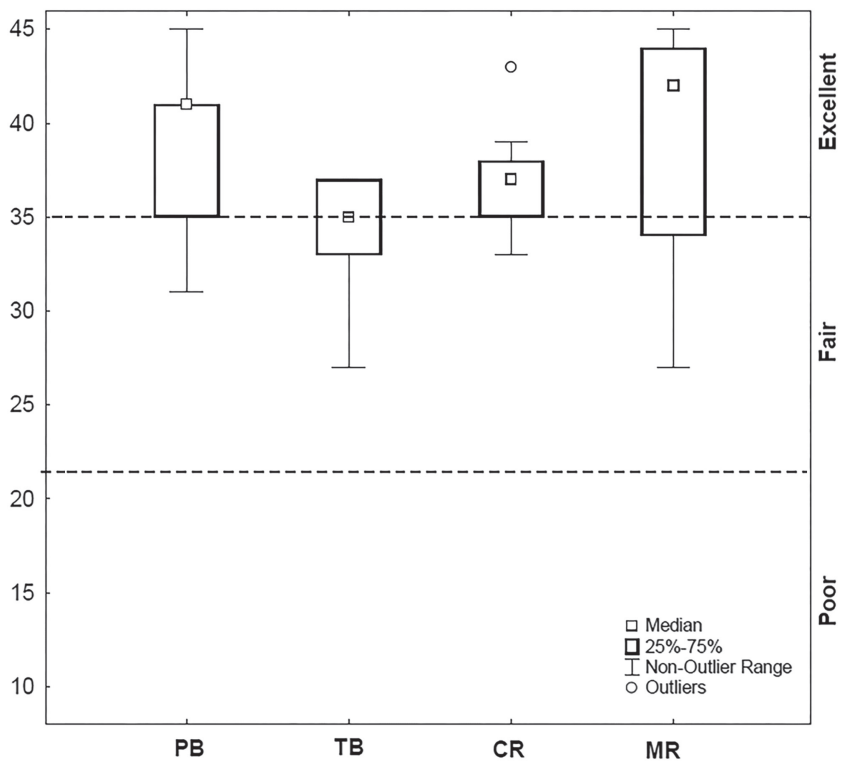

Fig. 2. Distribution of the $\mathrm{IBI}_{\mathrm{PNP}}$ in the strata sampled for the Pantanal National Park during the dry season (Oct-Nov, 2010 and 2011). PB - permanent bays, TB - temporary bays, $\mathrm{CR}$ - corixos, $\mathrm{MR}$ - main rivers.

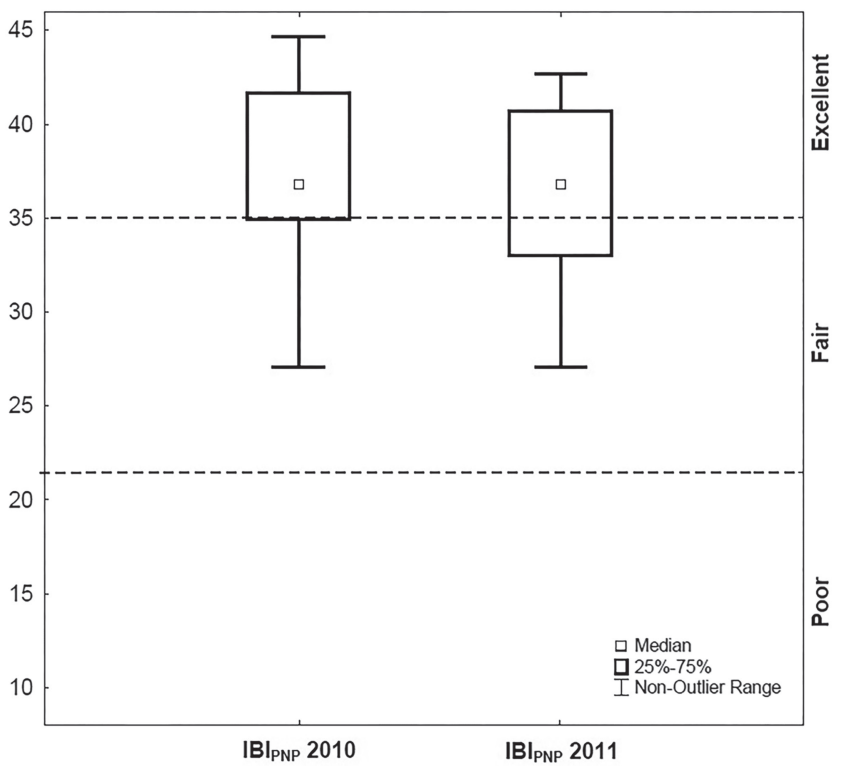

Fig. 3. Distribution of the $\mathrm{IBI}_{\mathrm{PNP}}$ for the years of 2010 and 2011. 


\section{Discussion}

The PNP is a large area within the Pantanal biome representing the wetlands of Central-West Brazil. This national park is closed for public visitation as of this publication. However, tourism will soon be allowed, which may result in additional sources of environmental impact if this activity is poorly managed. The building of small reservoirs in the headwaters of its main rivers may also lead to changes in the hydrological cycle and consequently modify the ecosystem dynamics. The multimetric indices are best suited to assess the impact on aquatic environments from multiple sources, as they take into account different ecological attributes of communities (Seegert, 2000). Thus, this attempt to conduct the first implementation of an index of biotic integrity to PNP is aimed to provide a monitoring tool and a reference condition for assessing future environmental impairment within the park and its surroundings.

Applying the IBI to a preserved area has the unique possibility of gathering information with confidence on the distribution of reference values. As the metrics are measured before the beginning of disturbance processes and virtually all sites can be considered in good or excellent biotic integrity, there is no need to model sample values for baseline conditions in this case. A logical difficulty, however, is that in the absence of human impacts, the $\mathrm{IBI}_{\mathrm{PNP}}$ values become difficulty to validate. Considering scenarios of strong disturbance, some responses are easily predicted, such as the reduction of species richness, migratory fishes, and intolerant species. On the other hand, predicting the responses in advance of these impacts may be difficult and challenging when the disturbances are subtle. Because the PNP is a conservation unit not severely impacted, small values for a metric distribution were not expected. Score 1 should represent clear evidence of human impact, but within the range of observations on hand, sites classified as 1 are considered as far as possible from the best potential condition (score 5) for a determined metric. This is expected due to natural variation, even when analyzing pristine environments (Gerritsen et al., 2003). The challenge of adapting an IBI to an undisturbed area is just as difficult as adapting it to a heavily disturbed one, where natural conditions are no longer available. In both cases, not all classes of disturbance-integrity are present, and the researchers have only a partial view of the range of possibilities for the metrics distribution.

Due to this limitation, the choice for candidate metrics on IBI's adapted for other environments was investigated and the appropriate metrics were selected based on the three established criteria: range test, responsiveness, and redundancy (Whittier et al. 2007, Petesse et al., 2014). The second criteria, for example, attempted to identify metrics that were sensitive to environmental conditions, expecting that these responses will exacerbate in the presence of anthropogenic impacts. In addition, following Gerritsen et al. (2003), the choice for the kind of metric scoring reflected the fact that the observed values will act as reference for future impacts within the region.

Significant differences among the types of water bodies and between years on the mean values of $\mathrm{IBI}_{\mathrm{PNP}}$ were not detected. This suggests that despite being clearly heterogeneous, the categories of water bodies analyzed have similar integrities, and these patterns do not change significantly from one year to another. In other applications of the IBI, temporal variability have been generally less important than spatial variability (Petesse et al., 2007; Casatti et al., 2009; Terra, Araújo, 2010; Ferreira, 2011), being the reason why Casatti et al. (2009) reinforced the importance of investing in the spatial variability instead of seasonality in early adaptations of multimetric indices. Terra, Araújo (2010), for instance, reported that seasonality had no effect on Reservoir Fish Assemblage Index (RFAI; an adaptation of IBI for reservoirs) in a transitional riverdam stretch of the Paraíba do Sul River that covers the Funil reservoir.

Considering this question, the current temporal study and sampling did not investigate the variation during the hydrological cycle (drought, flood, ebb, and flow), which 1 is the most important factor controlling community dynamics in the Pantanal. It was expected that large changes in fish communities during hydrological cycle would occur, but the similarity found in the $\mathrm{IBI}_{\mathrm{PNP}}$ between the dry seasons of 2010 and 2011 suggests that the fish populations are adapted to such phenomena. When year to year variability is not important, Karr et al. (1986) recommended that fish sampling should be conducted in a season that minimizes variation in fish community, due to discrete events such as fish recruitment. Several factors contribute to variations in the observed values for the IBI of a particular place and time because the index is entirely dependent on the composition and abundance of the collected fish population. Some pattern of movements and the collectors experience, for example, interfere decisively in the species and individuals sampled (e.g., larger or smaller) (Fore et al., 1994). This makes the multimetric indices behave as a random variable, adding a stochastic component upon the estimate of biotic integrity. In the Pantanal, recruitment takes place mainly during flood periods. As such, more variation in the community composition during flow and flood periods due to fish migrations and recruitment can be expected. Fish sampling is also less efficient during these seasons due to the higher water volume. In the dry season, the aquatic environments are more constrained, and sampling is more efficient, yielding a better characterization of fish communities (Junk et al., 1989; Junk, 2001). These facts provide us a better picture of how fish community structures are organized, which is a practical concern if the $\mathrm{IBI}_{\mathrm{PNP}}$ is established as a monitoring tool.

Nine out of the 34 candidate metrics that were selected were related to fish richness and composition (3 metrics), abundance (4 metrics), composition of feeding 
groups ( 1 metric), and indicators at the community levels (1 metric). Four metrics handle with the patterns observed at higher taxonomic levels such as order (total number of Siluriformes species or abundance of Characiformes and Gymnotiformes) or family (total number of Cichlidae species). As an advantage, these taxonomic levels are easily recognized by non-specialists. Other metrics, such as total number of individuals or number of species comprising $80 \%$ of total abundance, are easily calculated. For example, there is no need to determine each species when counting how many species comprise $80 \%$ of total population. In addition, Petesse et al. (2007) concluded that calculating the index in terms of fish biomass or fish numbers generates similar results in a previous application of a multimetric index to reservoirs. If the same applies for the $\mathrm{IBI}_{\mathrm{pNp}}$, the application in numbers would require less field effort because it would be impracticable to weigh the high numbers of fish captured in the field (mainly the small ones).

The $\mathrm{IBI}_{\mathrm{PNP}}$ values are subject to the environmental characteristics observed within the park. In this case, it should be considered that the type of gear employed tended to catch the fish living in shallow habitats $(<2 \mathrm{~m}$ depth). Thus, there is a need to standardize the sampling method for future comparisons.

By combining indicators into an overall multimetric index to summarize biological condition, it is reinforced that the presented results in metrics combination always carried to the same baseline: PNP sites can be considered as references. Although the $\mathrm{IBI}_{\mathrm{PNP}}$ can be used as a reference for future adaptations, further studies are needed on the biology of each species and how they interact with their habitats. It is also suggested that wetland areas outside of the park with evident signs of anthropogenic degradation be selected to validate our results and to provide new opportunities to test the $\mathrm{IBI}_{\mathrm{PNP}}$.

In addition, research that has the opportunity to investigate pristine areas should be encouraged and supported by governments and international organizations because information in these areas, even if already available, cannot be easily transferred to multimetric indices. Given that budget and time to conduct additional studies is always a limitation, in addition to considering the degree of human impact on aquatic environments, such information will be of great value to substantiate management decisions.

\section{Acknowledgments}

This study is a partial result of a Ph.D thesis of CNMP advised by MP-Jr and FCP at University of São Paulo, Brazil. It was financially supported by Instituto Chico Mendes de Conservação da Biodiversidade (ICMBio). The authors are grateful to Dra. Maria Letizia Petesse for her valuable comments on previous versions of this manuscript. We would like to thank Dr(s). José Augusto F. de Lima, Cláudio Oliveira, Fábio di Dário, Marina Loeb, Flávio C.T. Lima, Flávio Bockmann and Fernando Apone for helping us with fish identification; to IBAMA for fish collection license and $\operatorname{Dr}(\mathrm{s})$. Bruno F. Melo and Ricardo Britzke that helped us with the fish sampling and identification in the field. We would also like to thank Michael James Stablein of the University of Illinois Urbana-Champaign for his translation services and review of this work.

\section{References}

Araújo FG, Fichberg I, Pinto BCT, Peixoto MG. A preliminary index of biotic integrity for monitoring the condition of the rio Paraiba do Sul, Southeast Brazil. Environ Manage. 2003; 32(4):516-26.

Bozzetti M, Schulz UH. An index of biotic integrity based on fish assemblages for subtropical streams in southern Brazil. Hydrobiologia [serial on the Internet]. 2004; 529(1-3):133-44 Available from: http://dx.doi. org/10.1007/s10750-004-5738-6

Britski HA, Silimon KZS, Lopes BS. Peixes do Pantanal: Manual de identificação. 2nd ed. Brasília: Embrapa Informação Tecnológica; 2007.

Casatti L, Ferreira CP, Langeani F. A fish-based biotic integrity index for assessment of lowland streams in southeastern Brazil. Hydrobiologia. 2009; 623(1):173-89.

Eschmeyer WN, Fricke R, van der Laan R. (eds). Catalog of fishes: genera, species, references [internet]. San Francisco: California Academy of Science; 2017 [updated 2017 Aug 31]. Available from: http://researcharchive.calacademy.org/research/ ichthyology/catalog/fishcatmain.asp

Fausch KD, Lyons J, Karr JR, Angermeier PL. Fish communities as indicators of environmental degradation. Am Fish Soc Symp. 1990; 8:123-44.

Ferreira CP, Casatti L. Integridade biótica de um córrego na bacia do alto rio Paraná avaliada por meio da comunidade de peixes. Biota Neotrop. 2006; 6(3):1-25.

Ferreira FC. Adaptação do índice de assembleia de peixes em reservatórios (IAPR) às áreas das PCH's - usinas Batista e Jorda Flor, rio Turvo (SP). [PhD Thesis]. Rio Claro, SP: Universidade Estadual Paulista; 2011.

Fore LS, Karr JR, Conquest LL. Statistical properties of an index of biological integrity used to evaluate water resources. Can J Fish Aq Sci. 1994; 51(5):1077-87.

Ganasan V, Hughes RM. Application of an index of biological integrity (IBI) to fish assemblages of the rivers Khan and Kshipra (Madhya Pradesh), India. Freshwater Biol. 1998; 40(2):367-83.

Gerritsen J, Carlson RE, Dycus DL, Faulkner C, Gibson GR, Harcum J, Markowitz SA. Lake and reservoir bioassessment and biocriteria: Technical guidance document. US Environmental Protection Agency. 2003 [updated 2014 Oct]. Available from: http:www.epa.gov/owow/monitoring/tech/lakes.html

Hughes RM, Larsen DP, Omernik JM. Regional reference sites: a method for assessing stream potentials. Environ Manage. 1986; 10(5):629-35.

Hughes RM, Oberdoff T. Applications of IBI concepts and metric to waters outside the Unites State and Canada. In: Simon TP, editor. Assessing the sustainability and biological integrity of water resources using fish communities. Boca Raton: CRC Press; 1999. p.79-93. 
Instituto Brasileiro do Meio Ambiente e dos Recursos Naturais Renováveis (IBAMA). Plano de manejo do Parque Nacional do Pantanal Matogrossense. 2003 [updated 2013 Jun]. Available from: http://www.icmbio.gov.br/portal/images/stories/imgsunidades-conservacao/parna_matogrossense.pdf

Junk WJ, Bayley PB, Sparks RE. The flood pulse concept in riverfloodplain systems. Can Spec Publ Fish Aquat Sci. 1989; 106: 110-27.

Junk WJ. The flood pulse concept of large rivers: learning from the tropics. Verh Internat Verein Limnol. 2001; 27:3950-53.

Karr JR. Assessment of biotic integrity using fish communities. Fisheries. 1981; 6(6):21-27.

Karr JR, Fausch KD, Angermeier PL, Yant PR, Schlosser IJ. Assessing biological integrity in running waters: A method and its rationale. Ill Nat Hist Surv. 1986; special publication 5:1-28.

McCormick FH, Hughes RM, Kaufmann PR, Peck DV, Stoddard JL, Herlihy AT. Development of an index of biotic integrity for the mid-Atlantic highlands region. Trans Am Fish Soc [serial on the Internet]. 2001; 130(5):857-77. Available from: http:// dx.doi.org/10.1577/1548-8659(2001)130<0857:DOAIOB $>2.0$ $\mathrm{CO} ; 2$

McDonough TA, Hickman GD. Reservoir Fish Assemblage Index development: a tool for assessing ecological health in Tennessee Valley Authority impoundments. In: Simon TP, editor. Assessing the sustainability and Biological Integrity of water resources using fish communities. Boca Raton: CRC Press; 1999. p.523-40.

Ministério do Meio Ambiente (MMA). Sistema Nacional de Unidades de Conservação da Natureza. Lei Federal no 9.985, de 18 de julho de 2000. Brasília (DF): Diário Oficial da União; 2000.

Oliveira C, Avelino GS, Abe KT, Mariguela TC, Benine RC, Ortí $\mathrm{G}$ et al. Phylogenetic relationships within the speciose family Characidae (Teleostei: Ostariophysi: Characiformes) based on multilocus analysis and extensive ingroup sampling. BMC Evol Biol. 2011; 11:275-325. Available from: https://doi. org/10.1186/1471-2148-11-275

Pauly D. Anecdotes and the shifting baseline syndrome of fisheries. Trends Ecol Evol. 1995; 10(10):430.

Petesse ML, Petrere Júnior M, Agostinho, AA. Defining a fish bio-assessment tool to monitoring the biological condition of a cascade reservoirs system in tropical area. Ecol Eng. 2014; 69:139-150. Available from: http://dx.doi.org/10.1016/j. ecoleng.2014.03.070

Petesse ML, Petrere Júnior M, Spigolon RJ. Adaptation of the Reservoir Fish Assemblage Index (RFAI) for assessing the Barra Bonita Reservoir (São Paulo, Brazil). River Res Appl [serial on the Internet]. 2007; 23(6):595-612. Available from: http://dx.doi.org/10.1002/rra.1001
Polaz CNM. Caracterização da Ictiofauna e Aplicação do Índice de Integridade Biótica no Parque Nacional do Pantanal Matogrossense, Poconé, MT. [PhD Thesis on the Internet]. São Carlos: Universidade de São Paulo; 2013 [cited 2013 Aug 28]. Available from: Biblioteca digital Universidade de São Paulo. http://www.teses.usp.br/teses/disponiveis/18/18139/ tde-26082013-091018/pt-br.php

Polaz CNM, Melo BF, Britzke R, Resende EK, Machado FA, Lima JAF, Petrere Júnior M. Fishes from the Parque Nacional do Pantanal Matogrossense, upper Paraguai River basin, Brazil. Check List. 2014; 10(1):122-30.

Pont D, Hugueny B, Beier U, Goffaux D, Melcher A, Noble R, Rogers C, Roset N, Schmutz S. Assessing river biotic condition at a continental scale: a European approach using functional metrics and fish assemblage. J Appl Ecol [serial on the Internet]. 2006; 43(1):70-80. Available from: http://onlinelibrary.wiley. com/doi/10.1111/j.1365-2664.2005.01126.x/abstract

Roset N, Grenouillet G, Goffaux D, Pont D, Kestemont P. A review of existing fish assemblage indicators and methodologies. Fisheries Manag Ecol [serial on the Internet]. 2007; 14(6):393-405. Available from: http://dx.doi.org/10.1111/ j.1365-2400.2007.00589.x

Seegert G. Considerations regarding development of index of biotic integrity metrics for large rivers. Environ Sci Policy. 2000; 3(S1):99-106.

Simon TP, Sanders RE. Applying an index of biotic integrity based on great river fish communities: considerations in sampling and interpretation. In: Simon TP, editor. Assessing the sustainability and Biological Integrity of water resources using fish communities. Boca Raton: CRC Press; 1999. p.475-505.

Stoddard JL, Herlihy AT, Peck DV, Hughes RM, Whittier TR, Tarquinio E. A process for creating multimetric indices for large-scale aquatic surveys. J N Am Benthol Soc. 2008; 27(4):878-91.

Terra BF, Araújo FG. A preliminary fish assemblage index for a transitional river-reservoir system in southeastern Brazil. Ecol Indic. 2010; 11:874-81.

Whittier TR, Hughes RM, Stoddard JL, Lomnicky GA, Peck DV, Herlihy AT. A structured approach for developing indices of biotic integrity: three examples from streams and rivers in the Western USA. Trans Am Fish Soc. 2007; 136(3):718-35.

Zar JH. Biostatistical Analysis. 5th ed. New Jersey: Upper Saddle River; 2010.
Submitted April 11, 2017 Accepted August 09, 2017 by Paulo Pompeu 